\title{
The Art of Dying
}

I wear my hand-stitched cloak with pride And finish the look with a pointy hat. I hold my paintbrush like a wand And brew potions to poison my cancer And cast spells to banish sad thoughts.

I mould clay and smooth it into shapes. When the medicine failed me, I made my own liver like a crescent moon Infested with tumour

And smashed it to pieces with a hammer.

When my cancer mocks me,

I spit metaphors and stories at it And the pain subsides.

And when the time comes,

I will use my cloak to keep me warm And my paintbrush as a walking stick To keep me upright Until my last breath.

And I will be buried in one of my clay pots So that really, the only thing the cancer took Was my body

And when that died, the cancer died too

The silly thing.

\section{Madeleine Openshaw}

Corresponding to Madeleine Openshaw, Imperial College School of Medicine, South Kensington, London SW7 2AZ, United Kingdom; mco10@ic.ac.uk

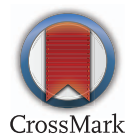

To cite Openshaw M. Med Humanit 2015;41:e6.

Med Humanit 2015;41:e6. doi:10.1136/medhum-2014-010653 